\title{
TRACKING AN OMNIDIRECTIONAL EVADER WITH A DIFFERENTIAL DRIVE ROBOT AT A BOUNDED VARIABLE DISTANCE
}

\author{
UbALdo RUIZ, Jose LUIS MARROQUIN, RAFAEL MURRIETA-CID \\ Center for Mathematical Research (CIMAT) \\ Jalisco S/N, Valenciana, Guanajuato, C.P. 36240, Mexico \\ e-mail: \{ubaldo, jlm, murrieta\}@cimat.mx
}

\begin{abstract}
In this paper, we address the pursuit-evasion problem of tracking an Omnidirectional Agent (OA) at a bounded variable distance using a Differential Drive Robot (DDR), in an Euclidean plane without obstacles. We assume that both players have bounded speeds, and that the DDR is faster than the evader, but due to its nonholonomic constraints it cannot change its motion direction instantaneously. Only a purely kinematic problem is considered, and any effect due to dynamic constraints (e.g., acceleration bounds) is neglected. We provide a criterion for partitioning the configuration space of the problem into two regions, so that in one of them the DDR is able to control the system, in the sense that, by applying a specific strategy (also provided), the DDR can achieve any inter-agent distance (within an error bound), regardless of the actions taken by the OA. Particular applications of these results include the capture of the OA by the DDR and maintaining surveillance of the $\mathrm{OA}$ at a bounded variable distance.
\end{abstract}

Keywords: pursuit-evasion, tracking, capturing, differential drive robot.

\section{Introduction}

In this paper, we address the pursuit-evasion problem of tracking an Omnidirectional Agent (OA) at a bounded variable distance using a Differential Drive Robot (DDR), on a Euclidean plane without obstacles. We assume that both players have bounded speeds, and that the DDR is faster than the evader, but due to its nonholonomic constraints it cannot change its motion direction instantaneously. Only a purely kinematic problem is considered, and any effect due to dynamic constraints (e.g., acceleration bounds) is neglected. We provide a criterion for partitioning the configuration space of the problem into two regions, so that in one of them the DDR is able to control the system, in the sense that, by applying a specific strategy (also provided), the DDR can achieve any inter-agent distance (within an error bound), regardless of the actions taken by the OA. Particular applications of these results include the capture of the OA by the DDR and maintaining surveillance of the OA at a bounded variable distance.

In our previous work (Murrieta-Cid et al., 2011), we presented a solution for the problem of tracking an omnidirectional mobile evader at a constant distance with a differential drive robot. In that paper, we obtained motion strategies for both the players and a long term solution for the game. The current work represents a generalization of the research presented there. In this paper, we provide conditions that establish whether or not it is possible for a DDR to track an OA at a bounded variable distance, and the DDR's motion strategies to perform the task. The pursuer's control objective is to reach an inter-player distance within an interval $\left[L_{G}-\epsilon, L_{G}+\epsilon\right]$, where $L_{G}$ is the goal distance for the pursuer, and $\epsilon$ is a prescribed small tolerance (determined by a positive real number); $\epsilon$ represents an overshoot or undershoot, due to the assumption that while one of the players changes the inter-player distance, the motion direction of the other player is unknown.

The main difference between the current work and that presented by Murrieta-Cid et al. (2011) is that previously we only provided a pursuer motion strategy that guarantees to maintain a constant distance between the players, while in this work we provide a pursuer motion policy that guarantees that it will be able to reach an inter-player distance within an interval $\left[L_{G}-\epsilon, L_{G}+\epsilon\right]$. This $L_{G}$ distance can be smaller or larger than the initial distance $L_{I}$ between the players.

We model the pursuer-evader system as a hybrid control problem combining two motion strategies. The 
first part of the pursuer's strategy is modeled continuously, and the goal is to maintain a constant distance between the players while the DDR aligns its heading with the evader's location (cf. Murrieta-Cid et al., 2011). The second part of the pursuer's strategy is modeled using a discrete system, in which the pursuer increases or decreases the inter-player distance. The DDR switches between both strategies until the desired condition (distance) is achieved.

In this work, we define a manifold over the space of parameters for the game, such that it induces a partition of this space into two disjoint regions. The DDR will be able to control the system whenever it is in one of those regions. By controlling we mean that the DDR can vary the inter-player distance freely. When the system is exactly on the manifold, no player controls it and this can be interpreted as a tied game. The motion strategies presented in this paper are applicable to several problems related to surveillance or capture:

- They allow a DDR to maintain an omnidirectional evader within a limited sensing range defined by a maximal $L_{\max }$ and a minimal $L_{\min }$ sensing distances.

- They allow a DDR to reduce the distance to the evader.

In the remainder of the paper, we describe the conditions that make the tasks listed above possible.

\section{Previous work}

A lot of work has been done in the area of pursuit-evasion games (Hájek, 1965; Isaacs, 1965; Başar and Olsder, 1982), and three main problems have received a lot of attention. They include finding, tracking and capturing a mobile evader with one or several pursuers.

In the finding problem (Isler et al., 2005; Hollinger et al., 2009), the objective is to establish some sort of visibility between the pursuer and the evader. In this case, the pursuer must sweep the environment so that the evader is not able to eventually sneak into an area that has already been explored. Deterministic (Suzuki and Yamashita, 1992; Guibas et al., 1999; Sachs et al., 2004; Tovar and LaValle, 2008) and probabilistic (Vidal et al., 2002; Hespanha et al., 2000; Chung, 2008) algorithms have been proposed to solve this problem.

In the capturing problem, the pursuer tries to get closer than a given distance $l$ to the evader. The goal of the evader is to keep the pursuer at all times farther from it than this capture distance. A classical problem is that of the homicidal chauffeur (Isaacs, 1965; Merz, 1971). In that game a faster pursuer (with respect to the evader) has as its objective to get closer than a given distance (the capture condition) to a slower but more agile evader, in order to run it over. The pursuer is a vehicle with a minimal turning radius. The game takes place in the Euclidean plane without obstacles, and the evader aims to avoid the capture condition. The problem tackled in this paper and its proposed solution are different to the homicidal chauffeur issue. Note that the change in the mechanical model for the pursuer (if this role is taken by the DDR, which can rotate in place) has as distinctive consequences that both the condition defining the winner and the motion strategies of the two players also change with respect to the homicidal chauffeur solution. Ruiz and Murrieta-Cid (2012) as well as Ruiz et al. (2013) presented time-optimal strategies for the game of capturing an omnidirectional evader with a differential drive robot. The results presented here, although not time-optimal, have the advantage over those of Ruiz and Murrieta-Cid (2012) as well as Ruiz et al. (2013) of allowing solution of two problems: capturing an OA evader with a DDR pursuer and maintaining surveillance at a bounded variable distance of an OA with a DDR.

In the tracking problem, the goal is maintaining visibility of the evader at all times, usually in an environment with obstacles (LaValle et al., 1997; González et al., 2002; Jung and Sukhatme, 2002; Bandyopadhyay et al., 2006; Bhattacharya and Hutchinson, 2010).

In recent years there has been a growing interest in related problems within the community of autonomous robots (Jung and Sukhatme, 2002; Kowalczuk and Czubenko, 2011), and specifically in robot motion planning (LaValle et al., 1997; González et al., 2002; Murrieta-Cid et al., 2007). LaValle et al. (1997) proposed game theory as a framework to formulate the tracking problem. Becker et al. (1995) presented an algorithm that operates by maximizing the probability of future visibility of the evader. This algorithm is also studied more formally by LaValle et al. (1997). Fabiani et al. (2002) present an approach that takes into account the positioning uncertainty of the robot pursuer.

The approach presented by Murrieta-Cid et al. (2005) computes a motion strategy by maximizing the shortest distance to escape, i.e., the shortest distance the evader needs to move in order to escape the pursuer's visibility region. González et al. (2002) propose a technique to track an evader without the need for a global map. Instead, a range sensor is used to construct a local map of the environment, and a combinatorial algorithm is then employed to compute a motion for the pursuer at each iteration. In our previous work (Murrieta-Cid et al., 2007) we specifically considered the case in which both the pursuer and the evader are omnidirectional; that led to a sufficient escape condition for the evader. Then (Murrieta-Cid et al., 2008) we again considered both players as omnidirectional systems moving in an 
environment containing obstacles. Further (Murrieta-Cid et al., 2008), we specifically addressed the combinatorial problem inherent to any strategy that considers visiting several locations in an environment with obstacles, and we provided a complexity result for this problem.

Bandyopadhyay et al. (2006) used a greedy approach for the problem of evading surveillance. To drive the greedy motion planning algorithm, a local minimum risk function is applied, called the vantage time. In the work of O'Kane (2008), a robot has to track an unpredictable target with bounded speed. The robot's sensors are manipulated to record general information about the target's movements and avoid the need for detailed, potentially damaging information about the target's position being available if the robot's sensors are accessed by other agent.

An interesting version of the problem involves multiple participants (several pursuers and evaders). Parker (2002) developed a method which attempts to minimize the total time in which the evaders escape surveillance. Jung and Sukhatme (2002) combined the application of mobile and static sensors. The authors used a metric for measuring the degree of occlusion, based on the average mean free path of a random line segment.

Pursuit-evasion can be used in a variety of applications. For example, Tekdas and Yang (2010) noticed the similarity between pursuit-evasion games and mobile routing for networking. Applying this similarity, they proposed motion planning algorithms for robotic routers to maintain connectivity between a mobile user and a base station. That work also includes a proof-of-concept implementation. Other possible interesting application of pursuit-evasion is the control of a team of robots (Skrzypczyk, 2005) with the goal of achieving a specific robot formation, for example, tracking the trajectory of an agent designed as the leader (Prodan et al., 2013).

Our problem consists in determining motion strategies to always maintain surveillance of the evader (assuming surveillance at the beginning of the game). The evader is under pursuer surveillance whenever it is at a bounded variable distance to the pursuer. It is interesting to analyze this case because commercially available sensors have upper and lower range limits. In particular, even in the absence of obstacles, if the evader is farther or closer than the sensor range, then its location is unknown, and the surveillance is broken. Our results are applicable to problems in which the pursuer is a wheeled mobile robot tracking a human evader. An example of those problems is monitoring children with mobile robots to prevent them straying out-with a prescribed area set by their guardian. The results can also be applied for capturing an evader, i.e., moving the pursuer closer than a given distance to the evader.

\section{Preliminaries}

3.1. System model. Figure 1 shows the geometric description of the system. In an Euclidean plane, the OA's position is represented by $\left(x_{e}, y_{e}\right)$ and the DDR's position by $\left(x_{p}, y_{p}\right)$. We will refer to the line segment connecting these positions as the (variable length) rod, using an analogy with the problem presented by Schwartz and Sharir (1983). The length $L$ of this rod corresponds to the distance between both players (it can be interpreted as the measure of a range sensor). The angle $\theta_{p}$ denotes the angle of the pursuer's wheels with respect to the $x$-axis, and $\phi$ represents the angle of the rod (sensor's orientation) with respect to the $x$-axis. $\psi$ corresponds to the motion direction of the evader.

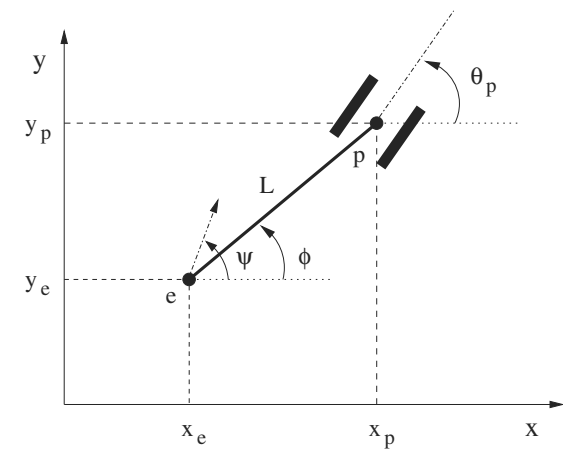

Fig. 1. Geometric model of the pursuer-evader system.

The evolution of the system is described by the following equations of motion:

$$
\begin{aligned}
& \dot{x}_{e}=u_{1} \cos u_{2}, \\
& \dot{y}_{e}=u_{1} \sin u_{2}, \\
& \dot{x}_{p}=u_{3} \cos \theta_{p}, \\
& \dot{y}_{p}=u_{3} \sin \theta_{p}, \\
& \dot{\dot{\theta}_{p}}=u_{4},
\end{aligned}
$$

where $u_{1} \in\left[0, V_{e}^{\max }\right]$ and $u_{2} \in[0,2 \pi)$ are the OA's controls, and they represent its speed and motion direction, respectively. Note that we assume that $u_{1}$ takes only positive values, but since $u_{2}$ takes any value between $[0,2 \pi)$ after a time $t$ the evader can reach any position inside or in the boundary of a circle with radius $u_{1} t$ centered at the evader's initial position. For the DDR pursuer, we use the usual assignment of control inputs (Balkcom and Mason, 2002). The DDR controls its linear velocity $u_{3} \in\left[-V_{p}^{\max }, V_{p}^{\max }\right]$ and the rate of change of its motion direction $u_{4}$.

3.2. Previous results. Previously (Murrieta-Cid et al., 2011) we presented the conditions under which it is possible for a differential drive robot (the pursuer) to track 
an omnidirectional mobile evader at a constant distance. To make this paper self-contained, we include here a brief review of the most important results in that work.

We have that the kinematic equations for a DDR (see LaValle, 2006) are given by

$$
\begin{aligned}
& u_{3}=V_{p}=\frac{\left(w_{r}(t)+w_{l}(t)\right) R}{2}, \\
& u_{4}=\dot{\theta}_{p}=\frac{\left(w_{r}(t)-w_{l}(t)\right) R}{2 b},
\end{aligned}
$$

where $u_{3}$ is the linear velocity, $u_{4}$ is its angular velocity, $w_{i}$ is the angular velocity of the $i$-th wheel, $R$ is the radius of the wheels, and $b$ is the distance between the center of the robot and the wheel location.

Without loss of generality, in what follows we will assume $R=1$. Adding and subtracting Eqns. (2) and (3) and solving for $u_{4}$, one obtains

$$
u_{4}=\frac{1}{b}\left(w_{r}-u_{3}\right)
$$

and

$$
u_{4}=\frac{1}{b}\left(-w_{l}+u_{3}\right) .
$$

These equations mean that for a given value of $w_{r}$ (resp. $w_{l}$ ) there is a linear relation between the controls $u_{3}$ and $u_{4}$

Recalling that $R=1$, the absolute value of the angular velocities $w_{r}, w_{l}$ is bounded by $V_{p}^{\max }$, the maximum attainable linear speed. The maximum counterclockwise turning speed $u_{4}^{\max }$ is obtained when either $w_{r}=V_{p}^{\max }$ or $w_{l}=-V_{p}^{\max }$. For these values, one may obtain from Eqn. (4) and (5) respectively

$$
0 \leq u_{4}^{\max }=\frac{1}{b}\left(V_{p}^{\max }-u_{3}\right)
$$

with

$$
u_{3}=\frac{1}{2}\left(V_{p}^{\max }+w_{l}\right) \geq 0,
$$

and

$$
0 \leq u_{4}^{\max }=\frac{1}{b}\left(V_{p}^{\max }+u_{3}\right)
$$

with

$$
u_{3}=\frac{1}{2}\left(w_{r}-V_{p}^{\max }\right) \leq 0 .
$$

Similarly, the maximum clockwise turning speed $u_{4}^{\min }$ is obtained when either $w_{r}=-V_{p}^{\max }$ or $w_{l}=$ $V_{p}^{\max }$, and one has from Eqns. (4) and (5)

$$
0 \geq u_{4}^{\min }=\frac{1}{b}\left(-V_{p}^{\max }-u_{3}\right)
$$

with

$$
u_{3}=\frac{1}{2}\left(-V_{p}^{\max }+w_{l}\right) \leq 0
$$

and

$$
0 \geq u_{4}^{\min }=\frac{1}{b}\left(-V_{p}^{\max }+u_{3}\right)
$$

with

$$
u_{3}=\frac{1}{2}\left(w_{r}+V_{p}^{\max }\right) \geq 0 .
$$

Equations (6), (8), (10) and (12) may be combined in the inequality

$$
\left|u_{4}\right|=\left|\dot{\theta}_{p}\right| \leq \frac{1}{b}\left(V_{p}^{\max }-\left|u_{3}\right|\right) .
$$

This inequality characterizes the space of valid controls (control space $\left(u_{3}, u_{4}\right)$ ) for the DDR, which corresponds to the boundary and interior of the rhombus depicted in Fig. 2 .

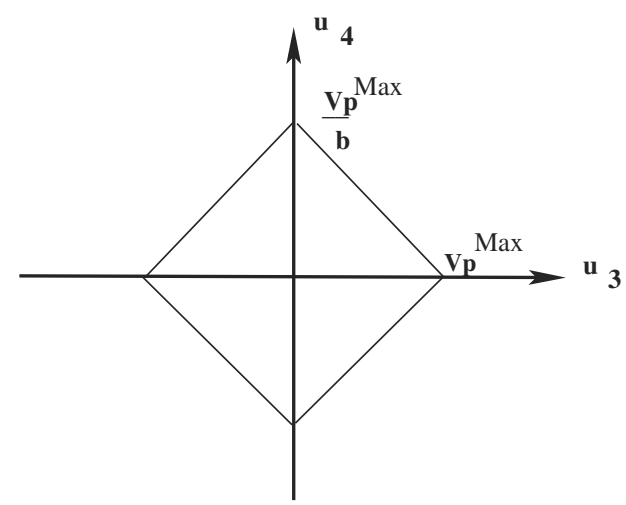

Fig. 2. Control space $\left(u_{3}, u_{4}\right)$.

The relation between the evader and the pursuer positions is given by

$$
\left(\begin{array}{l}
x_{p} \\
y_{p}
\end{array}\right)=\left(\begin{array}{l}
x_{e} \\
y_{e}
\end{array}\right)+L\left(\begin{array}{c}
\cos \phi \\
\sin \phi
\end{array}\right) .
$$

Computing the time derivative of Eqn. (15), and recalling that $\dot{L}=0$ (Murrieta-Cid et al., 2011), we proved that the linear speed $u_{3}^{*}$ of the pursuer required to maintain a constant distance $L$ to the evader is in fact fixed: for given values of $u_{1}, u_{2}, \theta_{p}$ and $\phi, u_{3}^{*}$ is given by

$$
u_{3}^{*}\left(\phi, \theta_{p}, u_{1}, u_{2}\right)=\frac{u_{1} \cos \left(u_{2}-\phi\right)}{\cos \left(\theta_{p}-\phi\right)} .
$$

The authors also proved that when the pursuer successfully tracks the evader to a constant distance (i.e., when $u_{3}=u_{3}^{*}$ ), the angular velocity of the $\operatorname{rod} \dot{\phi}$ is

$$
\dot{\phi}\left(\phi, \theta_{p}, u_{1}, u_{2}\right)=\frac{u_{1} \sin \left(\theta_{p}-u_{2}\right)}{L \cos \left(\theta_{p}-\phi\right)} .
$$

Evaluating the expression (14) for $u_{3}=u_{3}^{*}$, we get

$$
\max \left|\dot{\theta}_{p}\right|=\max \left|u_{4}\right|\left(u_{3}^{*}\right)=\frac{1}{b}\left(V_{p}^{\max }-\left|u_{3}^{*}\right|\right) .
$$


Equation (18) gives the maximum rate of rotation for the pursuer, given a specified linear speed $u_{3}^{*}$. In Eqns. (16) and 17) we can observe that as $\theta_{p}-\phi$ approaches $\pi / 2$ the required value for $u_{3}^{*}$ increases, requiring an infinite value at $\pi / 2$. As $\theta_{p}-\phi$ approaches zero, the required value of $u_{3}^{*}$ and hence $\dot{\phi}$ decrease. A pursuer with bounded speed must avoid the situation when $\theta_{p}-\phi \rightarrow$ $\pi / 2$, since at some point it will not be able to satisfy the required value for $u_{3}^{*}$ to maintain tracking at a constant distance. An antagonistic evader will therefore seek to lead the system to $\theta_{p}-\phi=\pi / 2$ to break surveillance. To this end, both players manipulate the change in $\theta_{p}-\phi$ given by $\dot{\phi}-\dot{\theta}_{p}$.

Note that the evader can control $\dot{\phi}$ directly using its controls $u_{1}$ and $u_{2}$ (Eqn. (17)), but also $\dot{\theta_{p}}$ indirectly, through $u_{3}^{*}$, since it can maximize the required linear velocity of the pursuer reducing the maximum feasible value for $u_{4}$ (see Eqn. (16).

Hence, the evader's optimal motion direction $u_{2}^{*}$ is the one that maximizes the difference $|\dot{\phi}|-\max \left|\dot{\theta}_{p}\right|$. The equation below establishes $u_{2}^{*}$ :

$$
u_{2}^{*}=\left\{\begin{array}{r}
\psi_{4}=\operatorname{atan}\left(\frac{-\sin \phi+\frac{b}{L} \cos \theta_{p}}{-\cos \phi-\frac{b}{L} \sin \theta_{p}}\right) \\
\text { if }\left(\theta_{p}-\phi\right) \in[0, \pi], \\
\psi_{3}=\operatorname{atan}\left(\frac{-\sin \phi-\frac{b}{L} \cos \theta_{p}}{-\cos \phi+\frac{b}{L} \sin \theta_{p}}\right) \\
\text { if }\left(\theta_{p}-\phi\right) \in(\pi, 2 \pi) .
\end{array}\right.
$$

The difference $|\dot{\phi}|-\max \left|\dot{\theta}_{p}\right|=0$ expressed as a function of the players' controls is given by

$$
\begin{aligned}
& \underbrace{\frac{\left|u_{1}^{*} \sin \left(\theta_{p}-u_{2}^{*}\right)\right|}{\left|L \cos \left(\theta_{p}-\phi\right)\right|}}_{|\dot{\phi}(t)|-\left|u_{4}^{* *}\right|=\max \left|\dot{\theta}_{p}(t)\right|}=0, \overbrace{\left.\left|\frac{u_{1}^{*} \cos \left(u_{2}^{*}-\phi\right)}{\cos \left(\theta_{p}-\phi\right)}\right|\right)}^{u_{3}^{* *}\left(u_{1}, u_{2}\right)}=0 \\
& \left.-\frac{1}{b} \mid V_{p}^{\max }\right)
\end{aligned}
$$

where $u_{1}^{*}$ is the maximal linear velocity of the evader, that is, $u_{1}^{*}=V_{e}^{\max }, u_{2}^{*}$ is defined by Eqn. (19), $u_{3}^{* *}$ denotes the pursuer's linear velocity that maintains the constant inter-player distance between the players whenever the evader applies controls $u_{1}^{*}$ and $u_{2}^{*}$, and $u_{4}^{* *}$ denotes the pursuer's maximal angular velocity that it can use whenever the evader applies controls $u_{1}^{*}$ and $u_{2}^{*}$.

Doing some algebraic manipulation of Eqn. (20), Murrieta-Cid et al. (2011) proved that the condition $|\dot{\phi}|-$ $\max \left|\dot{\theta_{p}}\right|=0$ can be written as

$$
V_{p}^{\max }\left(\left|\cos \left(\theta_{p}-\phi\right)\right|\right)=\left|u_{1}^{*}\right| g\left(\phi, \theta_{p}, u_{2}^{*}\right),
$$

where

$$
g\left(\phi, \theta_{p}, u_{2}^{*}\right)=\left\{\begin{array}{r}
-\cos \left(\phi-u_{2}^{*}\right)-\frac{b}{L} \sin \left(\theta_{p}-u_{2}^{*}\right) \\
\text { if }\left(\theta_{p}-\phi\right) \in[0, \pi], \\
-\cos \left(\phi-u_{2}^{*}\right)+\frac{b}{L} \sin \left(\theta_{p}-u_{2}^{*}\right) \\
\text { if }\left(\theta_{p}-\phi\right) \in(\pi, 2 \pi) .
\end{array}\right.
$$

We stress the fact that the evader's motion is not given a priori. In our previous work (Murrieta-Cid et al., 2011), we presented a worst-case analysis. We proved that if the evader uses at all times the controls that maximize the difference $|\dot{\phi}(t)|-\max \left|\dot{\theta}_{p}(t)\right|$, that is, $u_{1}^{*}=V_{e}^{\max }$ and $u_{2}^{*}$ defined by Eqn. (19), and this difference is still negative, then no evader's control will make the difference equal to or greater than zero. Hence, whenever this condition holds, the pursuer can maintain a constant distance to the evader and it can align its heading with the evader location in finite time regardless of the evader motion.

This main result was presented by Murrieta-Cid et al. (2011) defining the manifold

$$
\begin{aligned}
& M\left(V_{e}^{\max }, V_{p}^{\max }, \theta_{p}, \phi\right) \\
& \quad=\left|\dot{\phi}\left(u_{1}^{*}, u_{2}^{*}\right)\right|-\frac{1}{b}\left(V_{p}^{\max }-\left|u_{3}^{* *}\right|\right),
\end{aligned}
$$

exactly equivalent to

$$
\begin{aligned}
& M\left(V_{e}^{\max }, V_{p}^{\max }, L, \theta_{p}, \phi\right) \\
& \quad=V_{p}^{\max }\left(\left|\cos \left(\theta_{p}-\phi\right)\right|\right)-\left|u_{1}^{*}\right| g\left(\phi, \theta_{p}, u_{2}^{*}\right)=0 .
\end{aligned}
$$

The manifold $M\left(V_{e}^{\max }, V_{p}^{\max }, \theta_{p}, \phi\right)=0$ is thus crucial for determining the behavior of the system, since it divides the state space into two regions: one $(M>0)$ in which the evader can break the constant distance to the pursuer, and the other $(M<0)$ in which the pursuer can maintain the constant distance to the evader and align its heading with the evader's location. Note that at the moment the pursuer's heading reaches parallelism with the rod (the pursuer's heading is pointing to the evader's location): it is possible for the pursuer to keep this parallelism by applying $u_{4}=\dot{\theta}_{p}=\dot{\phi}$.

\section{Pursuit-evasion at a bounded variable distance}

In this section, we present the conditions and motion strategies for both players that allow one to relax the constant distance constraint as long as the configuration of the system remains in the initial region. 


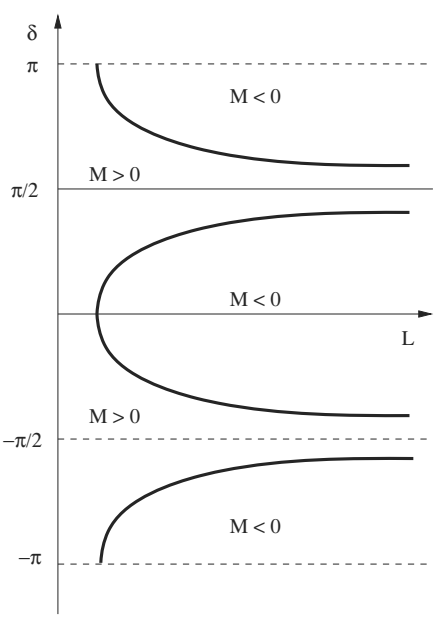

Fig. 3. Representation of $M(L, \delta)$ in the $(L, \delta)$ space.

For simplicity, instead of working with the variables $\left(V_{e}^{\max }, V_{p}^{\max }, L, \theta_{p}, \phi\right)$, we will use the space $(L, \delta)$ with

$$
\delta=\theta_{p}-\phi
$$

where $V_{e}^{\max }$ and $V_{p}^{\max }$ are fixed. In this case, $M\left(V_{e}^{\max }, V_{p}^{\max }, L, \theta_{p}, \phi\right)=0$ may be written as $M(L, \delta)=0$. We can rewrite Eqn. (24) as

$$
\begin{aligned}
& M\left(V_{e}^{\max }, V_{p}^{\max }, L, \theta_{p}, \phi\right) \\
& \quad=\left|u_{1}^{*}\right| g\left(\phi, \theta_{p}, u_{2}^{*}\right)-V_{p}^{\max }\left(\left|\cos \left(\theta_{p}-\phi\right)\right|\right)=0 .
\end{aligned}
$$

Using some trigonometric identities, in the proof of Lemma II in the work of Murrieta-Cid et al. (2011) it is shown that, if $\delta \in[0, \pi]$ (including $\delta \in[0, \pi / 2]$ ) and for $u_{2}^{*}$ as defined by Eqn. (19), we have

$$
\begin{aligned}
g\left(\phi, \theta_{p}, u_{2}^{*}\right) & =g\left(\phi, \theta_{p}\right) \\
& =\sqrt{1+\frac{2 b}{L} \sin \left(\theta_{p}-\phi\right)+\left(\frac{b}{L}\right)^{2}} .
\end{aligned}
$$

Substituting $g\left(\phi, \theta_{p}\right)$ given by Eqn. (27), $u_{1}^{*}=V_{e}^{\max }$ and Eqn. (25) into Eqn. (26), and recalling that $V_{e}^{\max }$ and $V_{p}^{\max }$ are fixed, we get

$$
\begin{aligned}
M(L, \delta)= & V_{e}^{\max } \sqrt{1+\frac{2 b}{L} \sin (\delta)+\left(\frac{b}{L}\right)^{2}} \\
& -V_{p}^{\max } \cos (\delta)=0 .
\end{aligned}
$$

Figure 3 shows the regions in the $(L, \delta)$ space where $M(L, \delta)>0$ or $M(L, \delta)<0$, and the curves (thick lines) where $M(L, \delta)=0$ for $\delta \in[-\pi, \pi]$.

In Fig. 3, we can observe that the value of $M(L, \delta)$ has some symmetry properties as the value of $\delta$ varies in $[-\pi, \pi]$. Using these properties, we have that the problem can always be reduced to the interval $[0, \pi / 2]$, the other quadrants being analogous.

Figure 4 shows the curve representing $M(L, \delta)=0$ in this interval. In the upper region of the figure are those configurations $(L, \delta)$ where the OA avoids constant distance tracking and $M(L, \delta)>0$. In the bottom region are those where the DDR maintains tracking and $M(L, \delta)<0$. The DDR "maintains tracking" in the region $M<0$, in the sense that, by applying the motion strategy provided below, the DDR can either maintain a constant distance to the evader or achieve any inter-agent distance $L_{G}$ (plus/minus a small value $\epsilon$ ), regardless of the actions taken by the evader.

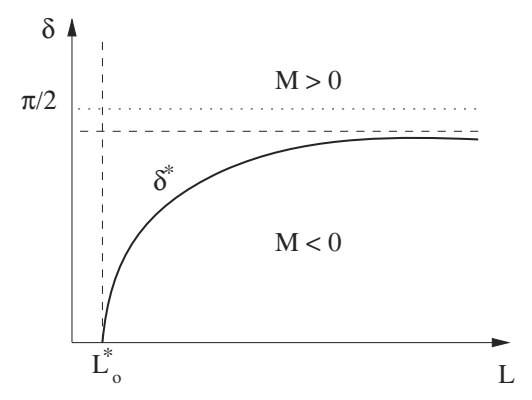

Fig. 4. Detail of Fig. 3, for $\delta \in[0, \pi / 2]$.

As our analysis will be based on the two regions composing the space $(L, \delta)$, it is important to prove some useful properties of the curve separating those regions.

Lemma 1. Let $\delta^{*}(L)$ be the curve separating the regions where $M(L, \delta)<0$ and $M(L, \delta)>0$.

1. There is a critical value $L=L_{o}^{*}$ such that $\delta^{*}\left(L_{o}\right)=$ 0 .

2. For $L>L_{o}^{*}, \delta^{*}(L)$ is a strictly increasing function.

3. If $L \rightarrow \infty$, then

$$
\delta^{*}(L) \rightarrow \cos ^{-1}\left(\frac{V_{e}^{\max }}{V_{p}^{\max }}\right) \leq \frac{\pi}{2} .
$$

4. For $L<\infty$,

$$
\delta^{*}(L)<\cos ^{-1}\left(\frac{V_{e}^{\max }}{V_{p}^{\max }}\right) \leq \frac{\pi}{2} .
$$

Proof. From Eqn. (28), and recalling that

$$
M\left(L, \delta^{*}(L)\right)=0,
$$

we have that

$$
\begin{aligned}
M\left(L, \delta^{*}(L)\right)= & V_{e}^{\max } \sqrt{1+\frac{2 b}{L} \sin \left(\delta^{*}(L)\right)+\left(\frac{b}{L}\right)^{2}} \\
& -V_{p}^{\max } \cos \left(\delta^{*}(L)\right)=0 .
\end{aligned}
$$


If $\delta^{*}(L)=0$, then

$$
M(L, 0)=V_{e}^{\max } \sqrt{1+\left(\frac{b}{L}\right)^{2}}-V_{p}^{\max }=0 .
$$

From the last expression, and by doing some algebra, we obtain the value $L_{o}$ such that $\delta^{*}\left(L_{o}\right)=0$ :

$$
L_{o}^{*}=\frac{V_{e}^{\max } b}{\sqrt{\left(V_{p}^{\max }-V_{e}^{\max }\right)\left(V_{p}^{\max }+V_{e}^{\max }\right)}},
$$

which may also be written as

$$
L_{o}^{*}=\frac{\rho b}{\sqrt{1-\rho^{2}}},
$$

where

$$
\rho=\frac{V_{e}^{\max }}{V_{p}^{\max }} .
$$

This proves the first part of the lemma.

Note that as $\rho \rightarrow 1, L_{o}^{*} \rightarrow \infty$, which implies that the OA evader can always break constant distance surveillance, which further means, as shown below, that the OA can always attain an arbitrary distance to the DDR pursuer. On the other hand, for $\rho \approx 0, L_{o}^{*} \rightarrow 0$. In what follows it will always be assumed that $\rho<1$.

From Eqn. (28), we observe that in order to keep a constant value of 0 for $M(L, \delta)$, if we increase the value of $L$, then we have also to increase the value of $\delta$. Therefore, $\delta^{*}(L)$ is a strictly increasing function with respect to $L>$ $L_{o}^{*}$, which proves the second part of the lemma. As $L \rightarrow$ $\infty$, we have that Eqn. (29) takes the form

$$
M\left(L, \delta^{*}(L)\right)=V_{e}^{\max }-V_{p}^{\max } \cos \left(\delta_{\infty}^{*}\right)=0 .
$$

By a straightforward manipulation of Eqn. (34), we obtain

$$
\delta_{\infty}^{*}=\cos ^{-1}(\rho) .
$$

Note that $\delta_{\infty}^{*}<\pi / 2$, and for $\rho \approx 0, \delta_{\infty}^{*} \rightarrow \pi / 2$. This proves the last part of the lemma.

This lemma implies that $\delta^{*}(L)$ is a bounded strictly increasing function with respect to the inter-player distance $L$. These properties allow us to define the regions in which each player controls the system. The DDR controls the system in the region $M<0$, in the sense that it can achieve any inter-agent distance $L \in\left[L_{G}-\right.$ $\left.\epsilon, L_{G}+\epsilon\right]$, regardless of the actions taken by the evader. The OA controls the system in the region $M>0$, in the sense that it can break the constant distance between the players, regardless of the actions taken by the pursuer.

Remark 1. From Lemma 1, we have that there is a critical value $L=L_{o}^{*}$ bounding $\delta^{*}(L)$ from the left. We have that

$$
L_{o}^{*}=\frac{\rho b}{\sqrt{1-\rho^{2}}},
$$

where

$$
\rho=\frac{V_{e}^{\max }}{V_{p}^{\max }} .
$$

In some cases $L_{o}^{*}<b$. The critical value corresponds to an inter-player distance located inside the robot's radius. In those cases, we must assume that the curve $\delta^{*}(L)$ is bounded by the critical value $L_{o}^{*}=b$, corresponding to configurations where the robot is in collision with the OA evader.

In what follows, we will show that from a given initial configuration $L_{I}, \delta_{I}$, the DDR (depending on the sign of $M\left(L_{I}, \delta_{I}\right)$ ) will be able to move in such a way that any desired inter-player distance $L_{G} \pm \epsilon$ (with certain restrictions) may be obtained in finite time.

4.1. DDR pursuer strategy. The following theorem establishes a strategy with which the DDR can reach a distance $L_{C} \in\left[L_{G}-\epsilon, L_{G}+\epsilon\right]$ (assuming $L_{G}-\epsilon \geq 0$ ) in finite time for any $\epsilon>0$, independently of the strategy followed by the OA.

Theorem 1. Assume that for the initial configuration $M\left(L_{I}, \delta_{I}\right)<0$. Given $\epsilon>0$, define $L_{B}^{*}=L_{o}^{*}+\epsilon>0$. Let $\left(L_{I}, L_{G}, L_{C}\right)>L_{B}^{*}+\epsilon$, be the initial, the goal and the current distance between the DDR pursuer and the $O A$ evader, respectively. The DDR can reach a distance $L_{C} \in$ $\left[L_{G}-\epsilon, L_{G}+\epsilon\right]$ in finite time, repeating the following strategy:

1. If $\delta\left(L_{C}\right)>0$, move at constant $L_{C}$, changing the DDR's heading until it is parallel to the orientation of the rod, i.e., make $\delta\left(L_{C}\right)=0$.

2. If $\delta\left(L_{C}\right)=0$, move during a time

$$
\widehat{T}=\min \left(T^{*}, \frac{\left|L_{C}-L_{G}\right|}{2 V_{p}^{\max }}\right)
$$

directly towards or away from the position of the $O A$ at time $t$, depending on the sign of $L_{C}-L_{G}$, with a velocity $V=\operatorname{sgn}\left(L_{C}-L_{G}\right) \cdot V_{p}^{\max }$, where

$$
T^{*}=\min \left(\frac{\epsilon}{2 V_{p}^{\max }}, \frac{L_{B}^{*} \sin \left(\delta^{*}\left(L_{B}^{*}\right)\right)}{V_{e}^{\max }}\right) .
$$

Proof. From the results of Murrieta-Cid et al. (2011) it follows directly that if $M\left(L_{C}, \delta\right)<0$, the DDR can yield $\delta\left(L_{C}\right)=0$ in finite time, using the controls $u_{3}^{*}, u_{4}^{*}$. For the second part of the strategy, one has to show that, if the DDR moves with a velocity $V=\operatorname{sgn}\left(L_{C}-L_{G}\right) \cdot V_{p}^{\max }$ during a time $\widehat{T},\left|L_{C}-L_{G}\right|$ will decrease and the system will remain in the region where $M\left(L_{C}, \delta\right)<0$. To do this, we consider the following two cases: 


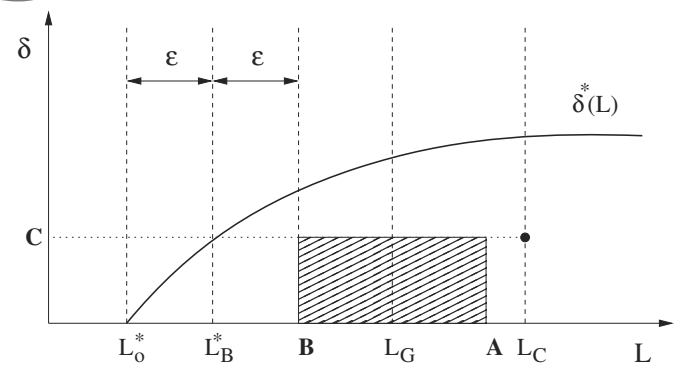

Fig. 5. Auxiliary constructions for the case $L_{G}<L_{C}$ (see the text).

Case I: $\left(L_{G}<L_{C}\right)$. In this case, we show that in the $(L, \delta)$ space, after an incremental motion of duration $\widehat{T}$, the new system configuration $\left(L_{C}^{\prime}, \delta^{\prime}\right)$ falls inside the shaded rectangle of Fig. 5 with $A=L_{C}-\left(V_{p}^{\max }-\right.$ $\left.V_{e}^{\max }\right) \widehat{T}, B=L_{B}^{*}+\epsilon$ and $C=\delta^{*}\left(L_{B}^{*}\right)$, which means that $L_{C}$ is decreasing as a function of time, and the system never leaves the region where $M(L, \delta)<0$.

Since the DDR wants to decrease the inter-player distance, it moves toward the OA during a time $\widehat{T}$. In order to show that $L_{C}^{\prime}<L_{C}$, it is enough to consider the worst case for the bound $A$, namely, when the OA moves directly away from the DDR at maximum speed, in which case $L_{C}^{\prime}=A<L_{C}$. We use analogous reasoning in order to prove the next cases.

To show that $L_{C}^{\prime} \geq B$, again one considers the worst case for this bound, namely, when the OA moves directly towards the DDR at maximum speed. In this case, from the definitions of $\widehat{T}$ and $T^{*}$, one has that

$$
\begin{aligned}
L_{C}^{\prime} & =L_{C}-\left(V_{p}^{\max }+V_{e}^{\max }\right) \widehat{T} \\
& >L_{C}-2 V_{p}^{\max } \widehat{T}>L_{C}-\epsilon>B .
\end{aligned}
$$

Finally, for the bound $C$, the worst case is obtained when the OA moves perpendicularly to the rod at maximum speed (see Fig. 6). In this case, the final angle $\delta^{\prime}$ satisfies

$$
\sin \left(\delta^{\prime}\right) \leq \frac{V_{e}^{\max } T^{*}}{L_{C}^{\prime}}<\frac{V_{e}^{\max } T^{*}}{L_{B}^{*}} \leq \sin \left(\delta^{*}\left(L_{B}^{*}\right)\right),
$$

where the last inequality follows from Eqn. (36). Since the $\sin (\cdot)$ function is increasing in the interval $[0, \pi / 2]$, from Lemma 1 one gets that $\delta^{\prime}<\delta^{*}\left(L_{B}^{*}\right)<\delta^{*}\left(L_{C}^{\prime}\right)$.

Case II: $\left(L_{G}>L_{C}\right)$. In this case there are only two bounds to consider for the new configuration $\left(L_{C}^{\prime}, \delta^{\prime}\right)$ (see Fig. 77: $D=L_{C}+\left(V_{p}^{\max }-V_{e}^{\max }\right) \widehat{T}$ and $C=\delta^{*}\left(L_{B}^{*}\right)$. The first one is obtained when the OA evader moves directly towards the DDR pursuer at maximum speed, in which case $L_{C}^{\prime}=D>L_{C}$, and the other when the DDR moves perpendicularly to the rod at time $t$, as in Case I, in

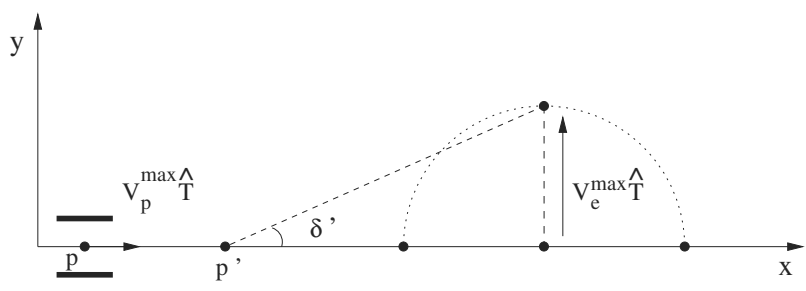

Fig. 6. DDR moves toward the OA, and the OA moves perpendicularly to the rod at maximum speed.

which

$$
\begin{aligned}
\sin \left(\delta^{\prime}\right) & \leq \frac{V_{e}^{\max } T^{*}}{L_{C}^{\prime}} \\
& <\sin \left(\delta^{*}\left(L_{B}^{*}\right)\right)<\sin \left(\delta^{*}\left(L_{C}^{\prime}\right)\right),
\end{aligned}
$$

and therefore $\delta^{\prime}<\delta^{*}\left(L_{C}^{\prime}\right)$ as above. This completes the proof.

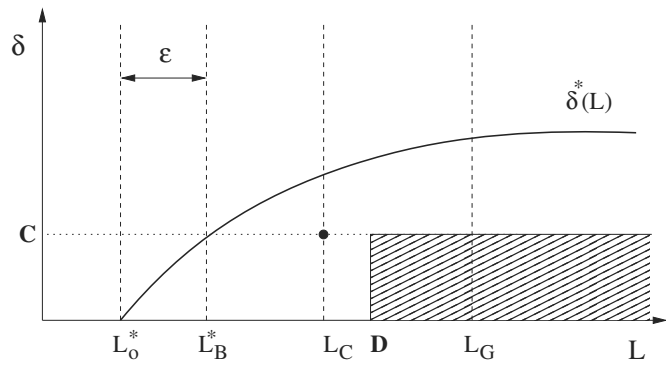

Fig. 7. Auxiliary constructions for the case $L_{G}>L_{C}$ (see the text).

Note that in both cases, since

$$
\widehat{T} \leq \frac{\epsilon}{2 V_{p}^{\max }}<\frac{\epsilon}{2 V_{e}^{\max }},
$$

the bound on the magnitude of the maximum overshoot (or undershoot) from the target distance is

$$
\left(V_{p}^{\max }+V_{e}^{\max }\right) \widehat{T}<2 V_{p}^{\max } \widehat{T} \leq \epsilon .
$$

Note also that this overshoot or undershoot is due to the assumption that while the pursuer changes the inter-player distance, the motion direction of the evader is unknown. In the worst case, the evader moves in the opposite direction to the one assumed to establish the bounds.

The theorem gives a constructive analysis which yields feasible motions for the DDR pursuer to accomplish its goals. Namely, to go from $L_{I}$ to $L_{G}$, the pursuer strategy is to alternate between two sub-strategies: (i) move at constant $L$ to make $\delta=0$ (which takes a finite amount of time), and (ii) move directly towards (or, away from) the evader for a finite time interval $\widehat{T}$. 
In Theorem 1 it is established that each time steps (i) and (ii) are applied, $\left|L_{I}-L_{G}\right|$ will decrease (or resp. increase) by a finite amount. This implies that the goal $\left|L_{I}-L_{G}\right| \leq \epsilon$ will be attained after a finite number of steps consisting in sub-steps (i) and (ii).

In the second part of the pursuer motion strategy, it moves a discrete time $\widehat{T}$. Thus, the number of sub-motions means the number of times the pursuer moves a finite time $\widehat{T}$ to achieve the desired inter-player distance.

Remark 2. In step (ii) of the proposed strategy, $L_{C}$ represents the inter-player distance at the instant where $\widehat{T}$ is computed. The strategy then dictates that the DDR moves during time $\widehat{T}$ at maximum speed in a direction that depends on the sign of $L_{C}-L_{G}$. During this time interval the instantaneous value of $L_{C}-L_{G}$ may change sign, but according to the proposed strategy this will not affect the DDR motion until $\widehat{T}$ is re-computed, which will not happen until the current incremental motion of step (ii) is completed, and if needed, $\delta\left(L_{C}\right)$ is made equal to zero using step (i). Once $L_{C}$ is inside the interval $L_{G} \pm \epsilon$, i.e., once the control goal has been achieved, depending on the application, it may be more convenient to change the strategy and apply only step (i) to maintain a constant inter-player distance $L_{G}^{\prime} \in\left[L_{G}-\epsilon, L_{G}+\epsilon\right]$.

In the theorem, $\epsilon$ is a parameter which represents the tolerance of reaching the desired inter-player distance. It allows one to determine a safety margin for not crossing the manifold defining the space partition (recall that the regions of the partition define the winner of the game).

Remark 3. On the one hand, a small $\epsilon$ allows reaching a distance that is closer to the desired inter-player distance $L_{G}$ between the players. But on the other hand, as $\epsilon$ decreases, the number of sub-motions necessary to reach a given configuration increases. Here $\epsilon$ may be set according to the user requirements, that is, the precision of the desired inter-player distance between the players versus the number of sub-motion (equivalent to the number of pursuer controls' switches) to reach a desired inter-player distance.

\subsection{Combining continuous and discrete modeling} for reaching $L_{C} \in\left[L_{G}-\epsilon, L_{G}+\epsilon\right]$. As mentioned above, we model the pursuer-evader system as a hybrid one combining continuous and discrete motion strategies.

The first part (continuous) of the pursuer's strategy is used to maintain a constant distance between the players and to align the pursuer's heading with the evader's location. Murrieta-Cid et al. (2011) proved that if $M<0$ then

$$
\left|\dot{\phi}\left(t, u_{1}^{*}, u_{2}^{*}\right)\right|<\max \left|\dot{\theta}_{p}\left(t, u_{3}^{* *}\right)\right|, \quad \forall t .
$$

Therefore, the pursuer aligns its heading with the evader's location in finite time. Recall that $\dot{\phi}$ represents the rate of change of $\phi$, the direction between the pursuer and evader locations, $u_{1}^{*}$ and $u_{2}^{*}$ denote respectively the evader's speed and the evader's motion direction that maximizes the difference $|\dot{\phi}|-\max \left|\dot{\theta}_{p}\right|$, and $u_{3}^{* *}$ denotes the pursuer's linear velocity that maintains a constant inter-player distance between the players whenever the evader applies controls $u_{1}^{*}$ and $u_{2}^{*}$.

Regarding the stability for the second (discrete) part of the pursuer's strategy, in which the pursuer increases or decreases the inter-player distance, consider the following.

An equilibrium point $x_{G} \in X$ is called Lyapunov stable if for any open neighborhood $O_{1}$ there exists another open neighborhood $O_{2}$ of $x_{G}$ such that $x_{I} \in O_{2}$ implies that $x(t) \in O_{1}$ for all $t>0$. If $X=\mathbb{R}^{n}$, an equivalent definition that is expressed in terms of the Euclidean metric is as follows: An equilibrium point $x_{G} \in \mathbb{R}^{n}$ is called Lyapunov stable if, for any $t>0$, there exists some $\lambda_{1}>0$ such that $\left\|x_{I}-x_{g}\right\|<\lambda_{1}$ implies that $\left\|x(t)-x_{G}\right\|<\lambda_{2}$ (see LaValle, 2006).

In the second part of the pursuer's strategy, the motions are executed in discrete time $\widehat{T}$. In Theorem 11. we prove that $\left|L_{C}-L_{G}\right|$ decreases and the system remains in the region $M(L, \delta)<0$. Hence, $L_{G} \pm \epsilon$ is reached in a finite number of sub-motions. There are direct equivalences between $\lambda_{1}$ and the region delimited by $M<0$, and also between $\lambda_{2}$ and $\epsilon$. Therefore, the system is stable.

\section{Simulations}

In this section, we present some simulations showing the players' strategies described before. The first simulation corresponds to the case when the DDR pursuer wants to reduce the inter-player distance. The initial parameters of the system are $V_{p}^{\max }=1 \mathrm{~m} / \mathrm{s}, V_{e}^{\max }=0.5 \mathrm{~m} / \mathrm{s}, b=1$ $\mathrm{m}, x_{e}=1 \mathrm{~m}, y_{e}=0 \mathrm{~m}, L=2 \mathrm{~m}, \theta_{p}=40^{\circ}, \phi=0^{\circ}$, $\epsilon=0.20, L_{o}=1 \mathrm{~m}$, and $L_{G}=1.25 \mathrm{~m}$.

Figure 8 shows the system trajectory in the space $(L, \delta)$. The trajectories followed by the players in the Euclidean plane are shown in Fig. 9 In this case, the DDR pursuer first aligns its heading with the rod's orientation, and then it moves directly towards the OA evader. The OA tries to move directly away from the DDR. We must point out the fact that in this example the DDR moves backwards, and the value of $u_{3}$ is negative.

In Fig. 10, we can observe the variation of the inter-player distance $L$ with respect to time, when the DDR wants to get closer to the OA. Initially, the DDR is aligning its heading with the rod orientation. During this time interval, the distance between both players remains constant. Once the DDR has aligned its heading, it starts moving toward the OA, while this player moves away from the DDR. Both players move in the same direction, but as the DDR is faster than the OA, the inter-player 


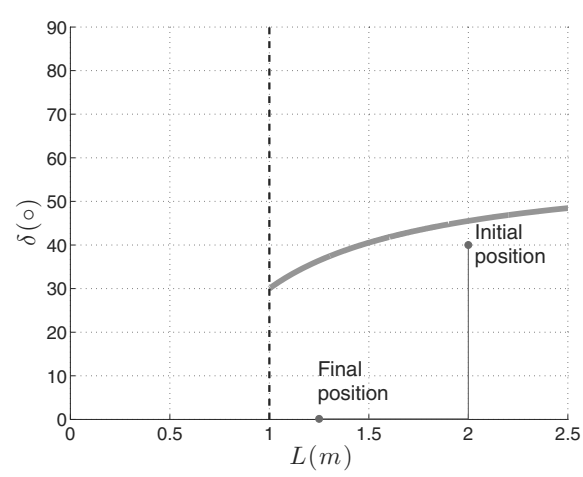

Fig. 8. Representation in $(L, \delta)$ of the case when the DDR decreases the inter-player distance. The thick curve corresponds to $M(L, \delta)=0$, and the thick dashed line to the value of $L_{B}$. The system is initially at the point $\left(2,40^{\circ}\right)$. The thin lines show the trajectory followed by the system. At the end, the system is at the point $\left(1.25,0^{\circ}\right)$.

distance decreases.

Figures 11 and 12 show the values of the evader's controls $u_{1}$ and $u_{2}$ during the game. From Fig. 11, we have that the evader moves always at maximum speed. In Fig. 12, and also in the trajectory in Fig. 9, we can note that, as part of the evader's strategy to escape from tracking at a constant distance, the evader is reducing the angle of its motion direction. From this simulation $u_{2}$ approaches a value close to $\pi / 2$. When the DDR aligns its heading with the evader's position and starts to move towards the evader, the evader also starts to move with $u_{2}=\phi+\pi$, trying to increase its distance to the DDR.

In Figs. 13 and 14, we show the pursuer's inputs $u_{3}$ and $u_{4}$. In Fig. 13, we can note how the value of $u_{3}$ necessary to maintain tracking at a constant distance is decreasing as the DDR aligns its heading with the evader's position. Once the DDR's heading is aligned, it moves at $u_{3}=V_{p}^{\max }$. Note that, as the heading is pointing in the opposite direction of the evader's position (see Fig. 9), the DDR moves backward to reduce the distance. In Fig. 14 the value of $u_{4}$ increases as the DDR aligns its heading with the evader's position. This behavior is explained by the fact that, as the value of $u_{3}$ is decreasing, the maximum feasible value of $u_{4}$ is increasing, and therefore the DDR is able to use a large value of $u_{4}$ as time elapses. Note that once the DDR's heading is parallel to the rod, the DDR stops its rotation and $u_{4}=0$.

In Fig. 15, we can observe how the required value of $\dot{\phi}$ is decreasing until the players switch to that part strategy. Note that for the second part of the strategy, in this simulation the players move following straight lines, and therefore $\dot{\phi}=0$. Figure 16 shows the variation of $\delta$ during the game.

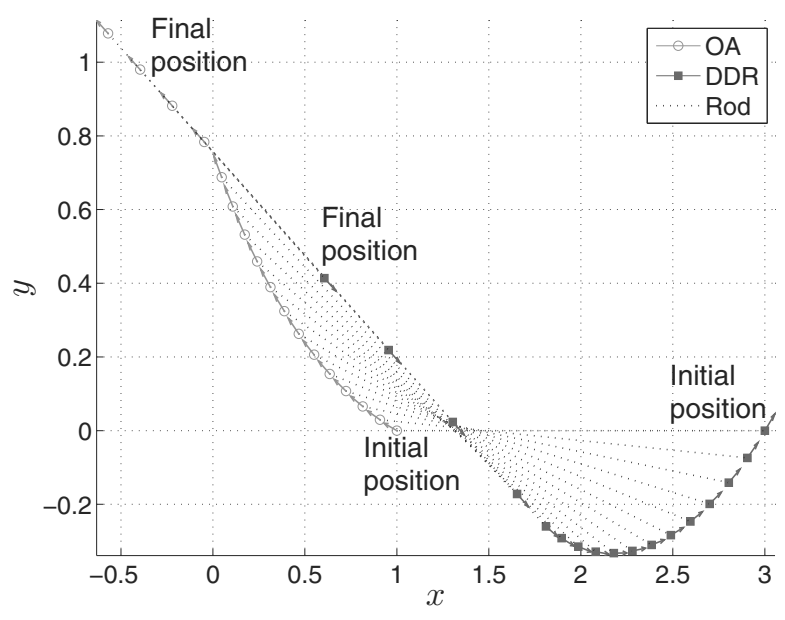

Fig. 9. Representation of the trajectories of the players corresponding to the system in Fig. 8 The DDR pursuer decreases the inter-player distance. The trajectories of the players were sub-sampled to show the motion direction of the players.

The second simulation corresponds to the case when the DDR pursuer increases the inter-player distance. The parameters are the same that in the first simulation, but with the goal distance $L_{G}=3.0 \mathrm{~m}$.

Figure 17 shows the system trajectory in the space $(L, \delta)$. The trajectories followed by the players in the Euclidean plane are shown in Fig. 18 The DDR pursuer first aligns its wheels with the rod's orientation, then it moves away from the evader, and the OA evader tries to get closer to the DDR.

In Fig. 19 we can observe the variation of the inter-player distance $L$ with respect to time, when the DDR gets farther to the OA.

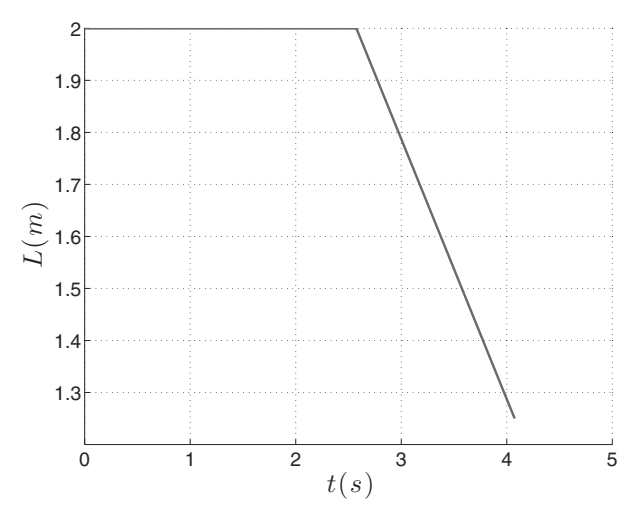

Fig. 10. Variation of $L$ as time elapses, corresponding to the system trajectory in Fig. 8 DDR decreases the interplayer distance. 


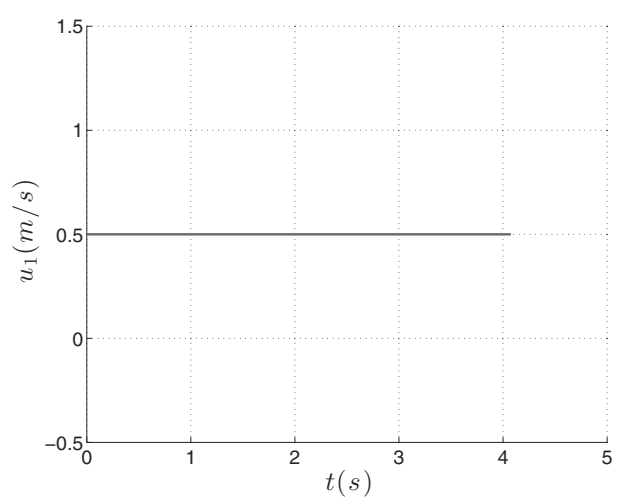

Fig. 11. Variation of the control input $u_{1}$, corresponding to the system trajectory of Fig. 9

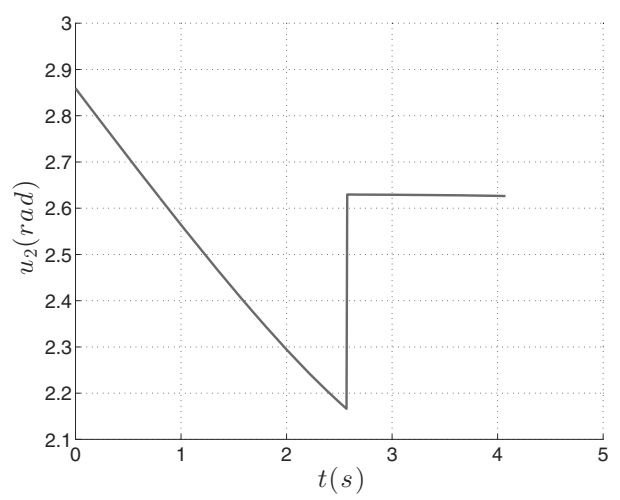

Fig. 12. Variation of the control input $u_{2}$, corresponding to the system trajectory of Fig. 9

Figures 20 and 21 show the values of the evader's controls $u_{1}$ and $u_{2}$ during the game. We can observe that, as in the previous simulation, the evader moves always at full speed. When the DDR aligns its heading with the evader's position and starts to move away the evader, the evader also starts to move with $u_{2}=\phi$, trying to decrease its distance to the DDR.

In Figs. 22 and 23, we show the pursuer's inputs $u_{3}$ and $u_{4}$. Also, as in the previous simulation, in both figures we can note that during the first part of the game the pursuer is changing the values of both inputs to align its heading with the position of the evader. Once the pursuer's heading is parallel to the evader's position, the pursuer moves at full linear speed $u_{3}$ towards the evader.

Figures 24 and 25 show the variation in $\dot{\phi}$ and $\delta$, respectively, during the game.

\section{Discussion and conclusions}

This work proposes an extension of the research presented by Murrieta-Cid et al. (2011). The motion strategies presented in this paper are applicable to several problems

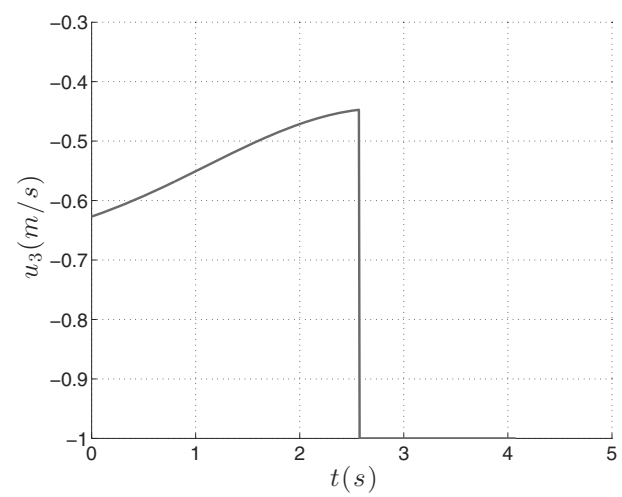

Fig. 13. Variation of the control input $u_{3}$, corresponding to the system trajectory of Fig. 9

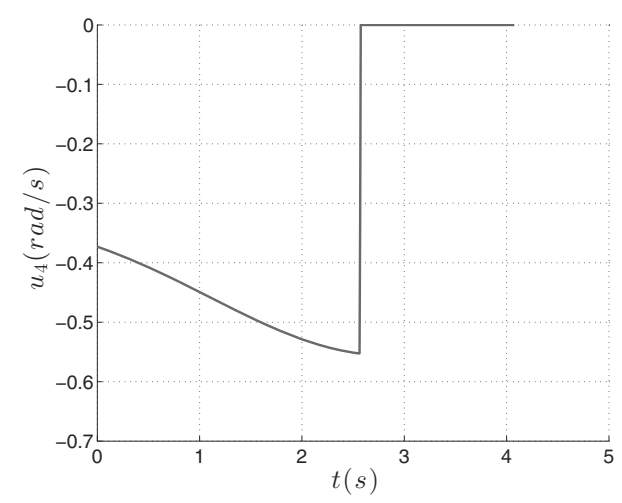

Fig. 14. Variation of the control input $u_{4}$, corresponding to the system trajectory of Fig. 9

related to surveillance or capture.

1. They allow a DDR pursuer to maintain an omnidirectional evader within a limited sensing range defined by a maximal $L_{\max }$ and a minimal $L_{\text {min }}$ sensing distances, provided that the limited sensing range satisfies the restriction imposed by $L_{\min }>L_{o}^{*}+2 \epsilon$.

2. They allow a DDR pursuer to reduce the distance to the omnidirectional evader, again provided that the desired inter-player distance satisfies the restriction $L_{G}>L_{o}^{*}+2 \epsilon$. Indeed, the problem of capturing an evader can be established in terms of this inter-player distance. That is, the capture condition is defined as moving the DDR closer than a given distance to the omnidirectional evader.

To our knowledge, this is the first time that a solution is proposed for both problems: tracking and capturing an omnidirectional evader with a differential drive robot. 


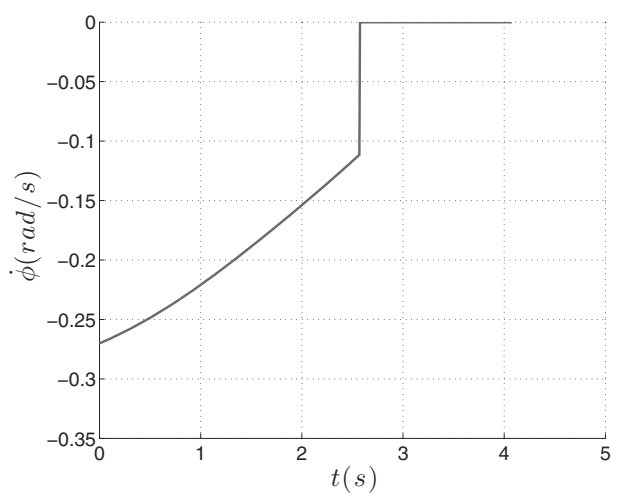

Fig. 15. Variation of $\dot{\phi}$, corresponding to the system trajectory of Fig. 9

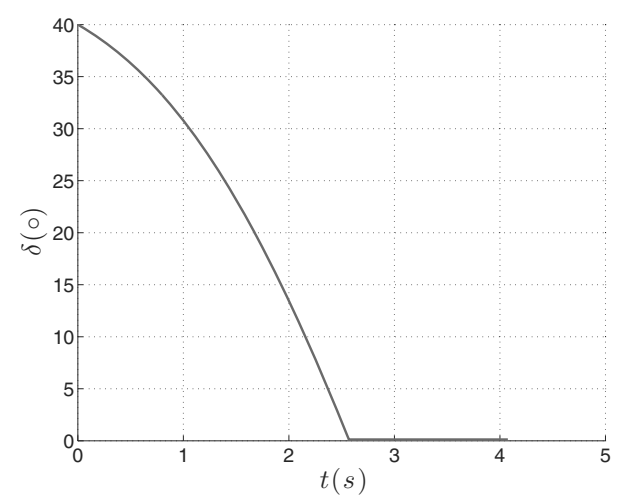

Fig. 16. Variation of the control input $\delta$, corresponding to the system trajectory of Fig. 9

It is important to stress that, if $M(L, \delta)<0$, then the DDR can obtain in finite time an inter-player distance $L \in\left[L_{G}-\epsilon, L_{G}+\epsilon\right]$, which satisfies $M(L, \delta)<0$, and such that $L_{G}>L_{o}^{*}+2 \epsilon$. In order to obtain the desired inter-player distance $L_{G}$ (within a tolerance $\epsilon$ ), the DDR performs the motion strategy described in Theorem 1 .

The main drawback of the motion strategies presented in this paper is that they are not necessarily optimal in time. However, note that the presented analysis gives constructive proofs which yield feasible motions for the players to obtain their goals in finite time.

In future work, we will consider acceleration bounds on the pursuer and the evader.

\section{References}

Başar, T. and Olsder, G. (1982). Dynamic Noncooperative Game Theory, Academic Press, New York, NY.

Balkcom, D. and Mason, M. (2002). Time optimal trajectories for bounded velocity differential drive vehicles, International Journal of Robotics Research 21(3): 219-232.

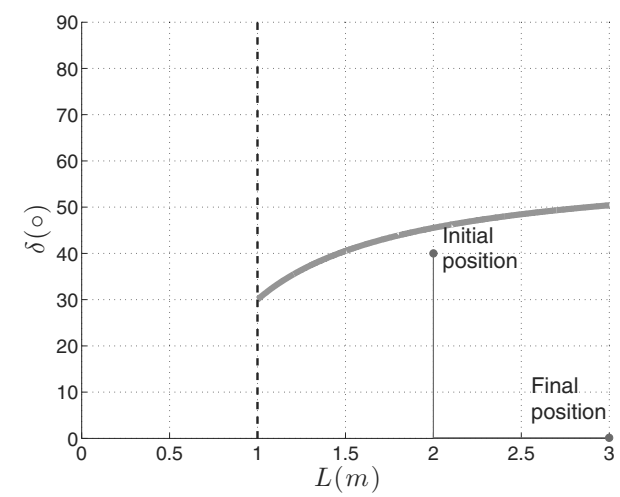

Fig. 17. Representation in $(L, \delta)$ of the case when the DDR increases the inter-player distance. The bold curve corresponds to $M(L, \delta)=0$, and the bold dashed line to the value of $L_{B}$. The system is initially at the point $\left(2,40^{\circ}\right)$. The thin lines show the trajectory followed by the system. At the end, the system is at the point $\left(3.0,0^{\circ}\right)$.

Bandyopadhyay, T., Li, Y., Ang, M. and Hsu, D. (2006). A greedy strategy for tracking a locally predictable target among obstacles, Proceedings of the International Conference on Robotics and Automation, ICRA 2006, Orlando, FL, USA, pp. 2342-2347.

Becker, C., González-Baños, H., Latombe, J. and Tomasi, C. (1995). An intelligent observer, Proceedings of the International Symposium on Experimental Robotics, ISER 1995, Stanford, CA, USA, pp. 153-160.

Bhattacharya, S. and Hutchinson, S. (2010). On the existence of Nash equilibrium for a two player pursuit-evasion game with visibility constraints, International Journal of Robotics Research 29(7): 831-839.

Chung, T. (2008). On probabilistic search decisions under searcher motion constraints, Proceedings of the International Workshop on the Algorithmic Foundations of Robotics, WAFR 2008, Guanajuato, Mexico, pp. 501-516.

Fabiani, P., González, H., Latombe, J. and Lin, D. (2002). Tracking an unpredictable target among occluding obstacles under localization uncertainties, Robotics and Autonomous Systems 38(1): 31-48.

González, H., Lee, C. Y. and Latombe, J. C. (2002). Real-time combinatorial tracking of a target moving unpredictably among obstacles, Proceedings of the International Conference on Robotics and Automation, ICRA 2002, Washington, DC, USA, pp. 1683-1690.

Guibas, L., Latombe, J., LaValle, S. M., Lin, D. and Motwani, R. (1999). A visibility-based pursuit-evasion problem, International Journal of Computational Geometry and Applications 9(4-5): 471-494.

Hájek, O. (1965). Pursuit Games, Academic Press, New York, NY. 


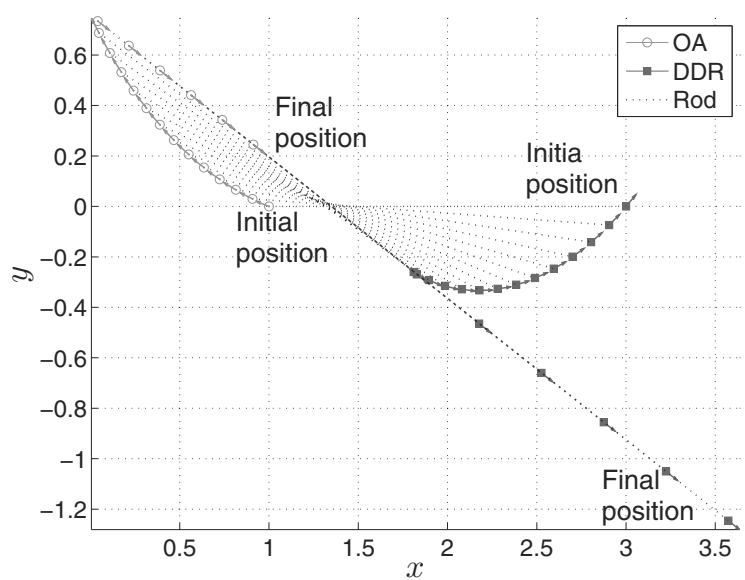

Fig. 18. DDR pursuer increases the inter-player distance. Again, the trajectories of the players were sub-sampled to show the motion direction of the players.

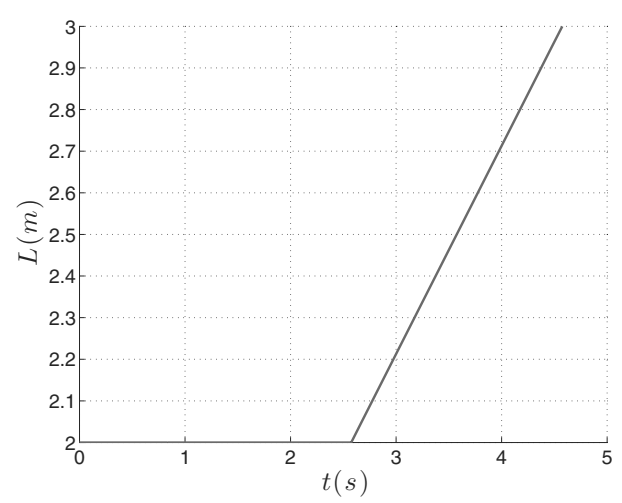

Fig. 19. Variation of $L$ as time elapses, corresponding to the system trajectory of Fig. 18 The pursuer increases the inter-player distance.

Hespanha, J., Prandini, M. and Sastry, S. (2000). Probabilistic pursuit-evasion games: A one-step Nash approach, Proceedings of the 39th International Conference on Decision and Control, Los Angeles, CA, USA, pp. 2272-2277.

Hollinger, G., Singh, S., Djugash, J. and Kehagias, A. (2009). Efficient multi-robot search for a moving target, International Journal of Robotics Research 28(2): 201-219.

Isaacs, R. (1965). Differential Games: A Mathematical Theory With Applications to Warfare and Pursuit, Control and Optimization, Academic Press, New York, NY.

Isler, V., Kannan, S. and Khanna, S. (2005). Randomized pursuit-evasion in a polygonal environment, IEEE Transactions on Robotics 21(5): 864-875.

Jung, B. and Sukhatme, G. (2002). Tracking targets using multiple robots: The effect of environment occlusion, $A u$ tonomous Robots 13(3): 191-205.

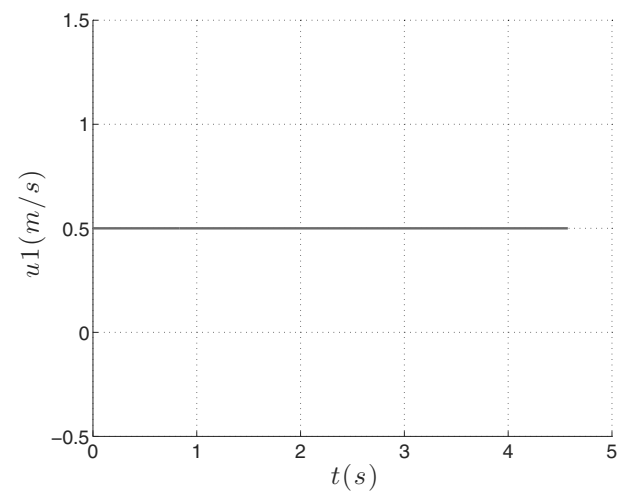

Fig. 20. Variation of the control input $u_{1}$, corresponding to the system trajectory of Fig. 18 .

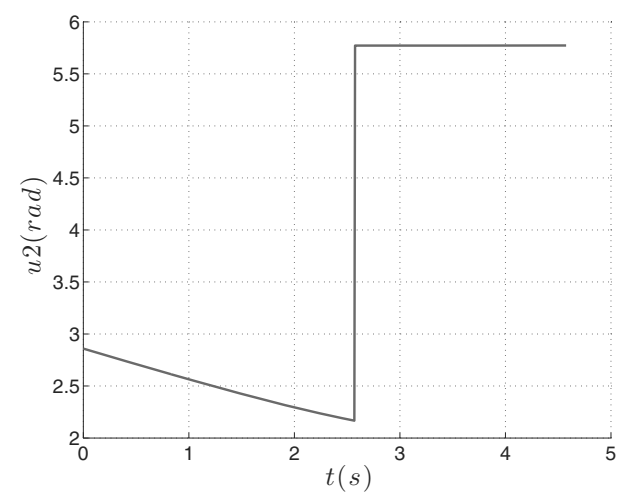

Fig. 21. Variation of the control input $u_{2}$, corresponding to the system trajectory of Fig. 18 .

Kowalczuk, Z. and Czubenko, M. (2011). Intelligent decision-making system for autonomus robots, International Journal and Applied Mathematics and Computer Science 21(4): 671-684, DOI: 10.2478/v10006-011-0053-7.

LaValle, S.M. (2006). Planning Algorithms, Cambridge University Press, New York, NY.

LaValle, S.M., González, H., Becker, C. and Latombe, J. (1997). Motion strategies for maintaining visibility of a moving target, Proceedings of the International Conference on Robotics and Automation, ICRA 1997, Albuquerque, NM, USA, pp. 731-736.

Merz, A. (1971). The Homicidal Chauffeur: A Differential Game, Ph.D. thesis, Stanford University, Stanford, CA.

Murrieta-Cid, R., Monroy, R., Hutchinson, S. and Laumond, J.-P. (2008). A complexity result for the pursuit-evasion game of maintaining visibility of a moving evader, Proceedings of the International Conference on Robotics and Automation, ICRA 2008, Pasadena, CA, USA, pp. 2657-2664.

Murrieta-Cid, R., Muppirala, T., Sarmiento, A., Bhattacharya, S. and Hutchinson, S. (2007). Surveillance strategies for a pursuer with finite sensor range, International Journal of Robotics Research 26(3): 233-252. 


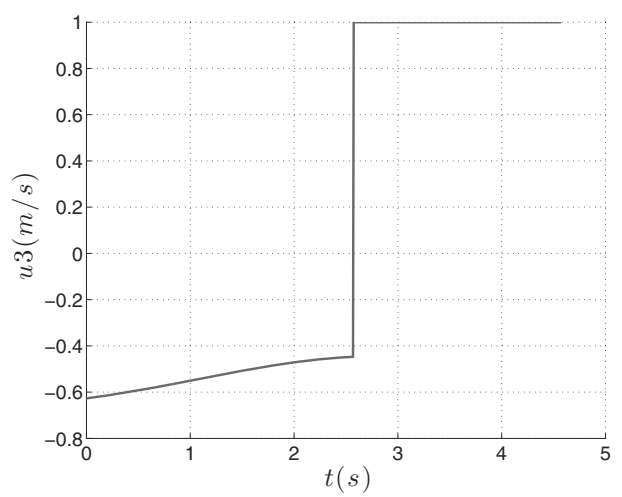

Fig. 22. Variation of the control input $u_{3}$, corresponding to the system trajectory of Fig. 18

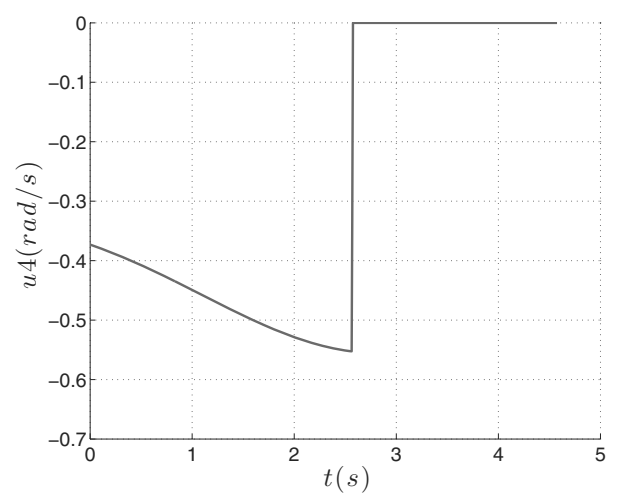

Fig. 23. Variation of the control input $u_{4}$, corresponding to the system trajectory of Fig. 18

Murrieta-Cid, R., Ruiz, U., Marroquin, J., Laumond, J. and Hutchinson, S. (2011). Tracking an omnidirectional evader with a differential drive robot, Autonomous Robots 31(4): 345-366.

Murrieta-Cid, R., Tovar, B. and Hutchinson, S. (2005). A sampling-based motion planning approach to maintain visibility of unpredictable targets, Autonomous Robots 19(3): 285-300.

O'Kane, J. (2008). On the value of ignorance: Balancing tracking and privacy using a two-bit sensor, Proceedings of the International Workshop on the Algorithmic Foundations of Robotics, WAFR 2008, Guanajuato, Mexico, pp. 235-249.

Parker, L. (2002). Distributed algorithms for multi-robot observation of multiple targets, Autonomous Robots 12(3): 231-255.

Prodan, I., Olaru, S., Stoica, C. and Niculescu, S.-I. (2013). Predictive control for trajectory tracking and decentralized navigation of multi-agent formations, International Journal of Applied Mathematics and Computer Science 23(1): 91-102, DOI: 10.2478/amcs-2013-0008.

Ruiz, U. and Murrieta-Cid, R. (2012). A homicidal differential drive robot, Proceedings of the International Conference

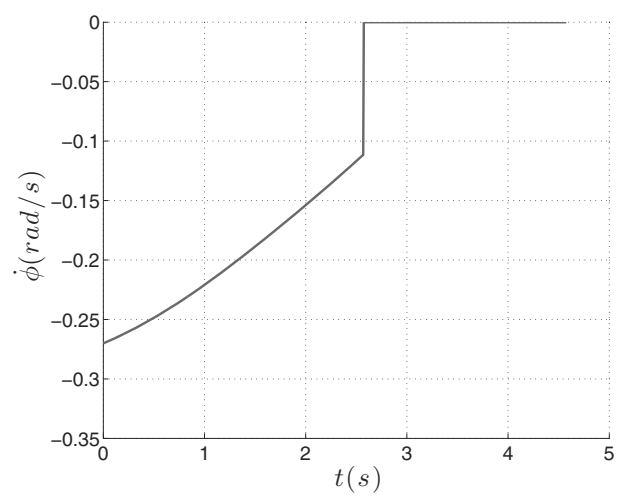

Fig. 24. Variation of $\dot{\phi}$, corresponding to the system trajectory of Fig. 18

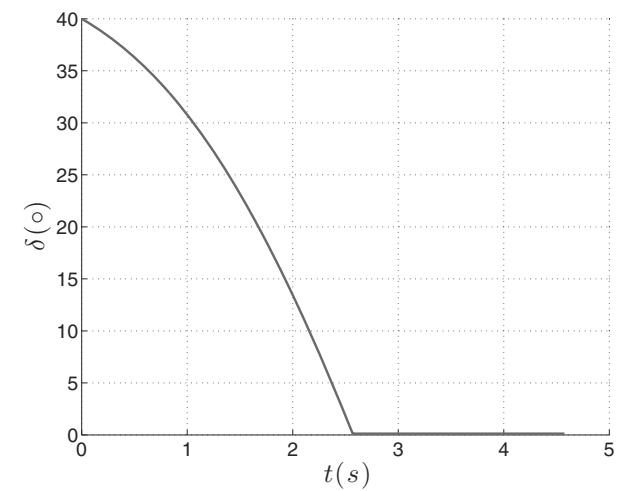

Fig. 25. Variation of the control input $\delta$, corresponding to the system trajectory of Fig. 18

on Robotics and Automation, ICRA 2012, St. Paul, MN, USA, pp. 3218-3225.

Ruiz, U., Murrieta-Cid, R. and Marroquin, J. (2013). Time-optimal motion strategies for capturing an omnidirectional evader using a differential drive robot, IEEE Transactions on Robotics 29(5): 1180-1196.

Sachs, S., LaValle, S. and Rajko, S. (2004). Visibility-based pursuit-evasion in an unknown planar environment, International Journal of Robotics Research 23(1): 3-26.

Schwartz, J.T. and Sharir, M. (1983). On the piano movers' problem. I: The case of a two-dimensional rigid polygon body moving amidst polygonal barriers, Communications on Pure and Applied Mathematics 36(3): 345-398.

Skrzypczyk, K. (2005). Control of a team of mobile robots based on non-cooperative equilibria with partial coordination, International Journal of Applied Mathematics and Computer Science 15(1): 89-97.

Suzuki, I. and Yamashita, M. (1992). Searching for a mobile intruder in a polygonal region, SIAM Journal on Computing 21(5): 863-888.

Tekdas, O. and Yang, W.and Isler, V. (2010). Robotic routers: Algorithms and implementation, International Journal of Robotics Research 29(1): 110-126. 
Tovar, B. and LaValle, S. (2008). Visibility-based pursuit-evasion with bounded speed, International Journal of Robotics Research 27(11-12): 1350-1360.

Vidal, R., Shakernia, O., Jin, H., Hyunchul, D. and Sastry, S. (2002). Probabilistic pursuit-evasion games: Theory, implementation, and experimental evaluation, IEEE Transactions on Robotics and Automation 18(5): 662-669.

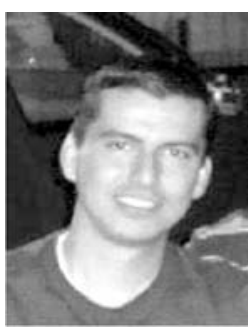

Ubaldo Ruiz received the B.Sc. degree in physics and mathematics (2005) and the M.Sc. degree in electrical engineering (2008), both from the University of Michoacan (UMSNH). In 2013 he received a Ph.D. in computer science from the Center for Mathematical Research (CIMAT), Guanajuato, Mexico. He is mainly interested in motion planning, differential games and optimal control.

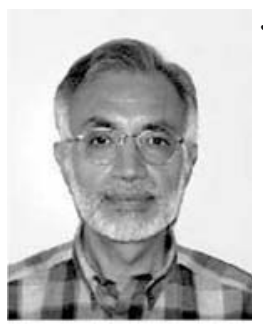

Jose Luis Marroquin did his Bachelor's degree at the School of Chemistry of the National Autonomous University of Mexico (UNAM), where he got his diploma in chemical engineering in 1968. He did his Master's and Ph.D. degrees at the Massachusetts Institute of Technology (MIT). He worked at PEMEX (Mexican Petroleum), in the areas of operations research (programming, mathematics and simulation), data visualization and processing of geophysics information. Since 1986 he has been working at the Center for Mathematical Research (CIMAT) in Guanajuato, Mexico, in which he is a senior researcher (Investigador Titular) and the head of the Computer Science Department. His main interests are image processing, computer vision and automatic learning, fields in which he has published around 100 papers and 2 books. He is an associate editor of Pattern Recognition and Computacion y Sistemas. He is a member of the Mexican National System of Researchers, level III

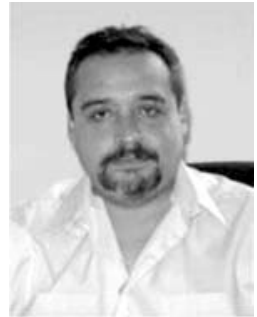

Rafael Murrieta-Cid received the B.S. degree in physics engineering from the Monterrey Institute of Technology and Higher Education, Monterrey, Mexico, in 1990, and the Ph.D. degree from National Polytechnic Institute, Toulouse, France, in 1998. His Ph.D. research was done with the Robotics and Artificial Intelligence Group of LAAS/CNRS. In 1998-1999, he was a postdoctoral researcher with the Department of Computer Science, Stanford University, CA, USA. In 2002-2004, he was a postdoctoral research associate with the Beckman Institute and the Department of Electrical and Computer Engineering, University of Illinois at Urbana-Champaign, Urbana, IL, USA From 2004 to 2006, he was a professor and the director of the Mechatronics Research Center at the Monterrey Institute of Technology and Higher Education (ITESM-CEM). Since March 2006, he has been with the Mathematical Computing Group, Center for Mathematical Research (CIMAT), Guanajuato, Mexico. His research interests include robotics and robot motion planning. He has published more than 50 papers in journals and international conferences on these topics.

Received: 25 April 2013

Revised: 9 November 2013

Re-revised: 18 January 2014 